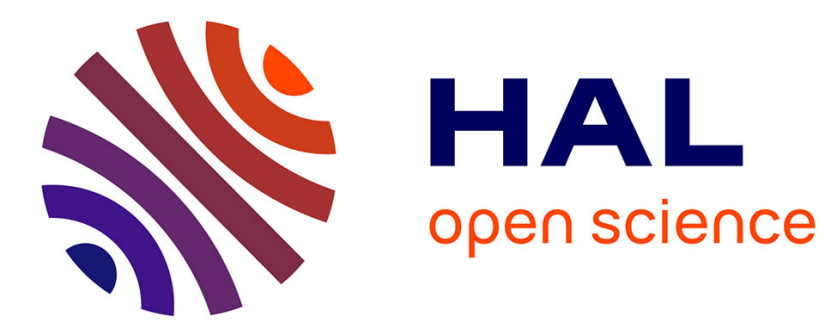

\title{
Perturbation of the Heat Lateral Diffusion by Interface Resistance in Layered Structures
}

Christian Frétigny, Jean-Yves Duquesne, D. Fournier

\section{To cite this version:}

Christian Frétigny, Jean-Yves Duquesne, D. Fournier. Perturbation of the Heat Lateral Diffusion by Interface Resistance in Layered Structures. International Journal of Thermophysics, 2015, 36 (5-6), pp.1281-1288. 10.1007/s10765-014-1648-7 . hal-01233797

\section{HAL Id: hal-01233797 \\ https://hal.science/hal-01233797}

Submitted on 28 Apr 2020

HAL is a multi-disciplinary open access archive for the deposit and dissemination of scientific research documents, whether they are published or not. The documents may come from teaching and research institutions in France or abroad, or from public or private research centers.
L'archive ouverte pluridisciplinaire HAL, est destinée au dépôt et à la diffusion de documents scientifiques de niveau recherche, publiés ou non, émanant des établissements d'enseignement et de recherche français ou étrangers, des laboratoires publics ou privés. 


\title{
Perturbation of the Heat Lateral Diffusion by Interface Resistance in Layered Structures
}

\author{
C. Frétigny · J.-Y. Duquesne · D. Fournier
}

Received: 16 December 2013 / Accepted: 3 June 2014

(C) Springer Science+Business Media New York 2014

\begin{abstract}
It is well established that interface resistances do usually exist in layered structures, and their values strongly depend on their origin. They may arise from different vibrational properties of the layers, nonharmonic processes at the interface, surface chemical contamination, interfacial defects, etc. Numerous studies have been published to evaluate their values, most of the time, in a perpendicular heat diffusion scheme. In this paper, the effect of interface resistances on the lateral modulated surface temperature of a layered structure for cylindrical symmetry heat diffusion is studied. The thermoreflectance microscope is a particularly convenient tool to record heat lateral diffusion from a surface modulated heated point and thus to evidence the presence of such resistance interfaces. In a first part, the theoretical model of heat diffusion in cylindrical symmetry, in a layered structure exhibiting an interface resistance between the layer and the substrate, is briefly described. In a second part, the $\mathrm{C} / \mathrm{I}$ configuration (good conductive layer deposited on an insulating substrate, with an interface resistance) is investigated. Experimental results illustrate the theory. In the third part, the reverse case I/C (insulating layer deposited on a conductive substrate, with an interface resistance) is discussed. To conclude, all the cases and the ability of the lateral diffusion to recover interface thermal resistances are compared.
\end{abstract}

Keywords Interface resistance - Thermal conductivity · Thermal diffusivity · Thermoreflectance microscopy

C. Frétigny

Laboratoire SIMM, UMR 7615, CNRS, UPMC, ESPCI, 10 rue Vauquelin, 75005 Paris, France

J.-Y. Duquesne, D. Fournier ( $\square)$

Institut des NanoSciences de Paris, UMR 7588, CNRS, UPMC, 4 place Jussieu, 75005 Paris, France

e-mail: daniele.fournier@upmc.fr 


\section{Introduction}

It is well established that interface resistances are most of the time present in layered structures. Their values are strongly dependant on their origin. They are smaller in the case of a crystalline lattice difference (case of super lattices deposited on semiconductors) than in the case of microstructure differences/nature of the two layers [1,2]. These interface resistances perturb the heat diffusion in the two directions, parallel and perpendicular. Numerous studies have been published to take into account their presence, most of the time, in a perpendicular heat diffusion scheme [3,4]. The 3 omega method [5], for instance, is particularly sensitive to these interface resistances. Most of the time, several samples with different thicknesses have to be studied. With the hypothesis of the same interface resistance for all the samples, it is straightforward to find the value of the interface resistance. In this paper, we study the effect of these interface resistances on heat lateral diffusion in the case of heat-modulated diffusion and show that lateral heat diffusion could be a particularly convenient tool to evidence the presence of such resistance interfaces. In a first part, we describe briefly the theoretical model of 3D heat diffusion in a layered structure and introduce the case of an interface resistance between the layer and the substrate. In a second part, we demonstrate the influence of the interface resistance for lateral heat diffusion in the case of a good conductive layer deposited on an insulator substrate. Experimental results illustrate the theory. In the third part, the reverse case (insulator deposited on a good conductive layer with an interface resistance) is discussed.

\section{Theoretical Approach}

In order to generate heat lateral diffusion, the size of the heat source has to be small compared to the thermal diffusion length. Thermoreflectance microscopy [6-8] is one of the setups using a small heat source. In this experiment, the sample is illuminated with an intensity modulated focused pump beam (less than $1 \mu \mathrm{m}$ diameter). Light being totally or partially absorbed, the light power is converted into heat which diffuses around the source. The spatio-temporal temperature field is detected at the surface of the sample with a second laser beam (probe beam) which is reflected by the heated surface. Lateral scans allow following lateral heat diffusion at $50 \mu \mathrm{m}$ from the heat source.

In the case of a semi-infinite bulk sample (thermal conductivity $k_{0}\left(\mathrm{~W} \cdot \mathrm{m}^{-1} \cdot \mathrm{K}^{-1}\right)$, thermal diffusivity $D_{0}\left(\mathrm{~m}^{2} \cdot \mathrm{s}^{-1}\right)$, the $3 \mathrm{D}$ modulated surface temperature in real space is obtained after the integration of [9]

$$
T(r, 0, \omega)=\int_{0}^{\infty} \frac{Q}{k_{0} \sigma_{0}} \exp \left(-\frac{\lambda^{2} d^{2}}{32}\right) J_{0}(\lambda r) \lambda \mathrm{d} \lambda,
$$

where $Q$ is the pump power (W) absorbed by the surface sample, $d$ is the diameter at $1 / \mathrm{e}^{2}$ of the Gaussian pump beam, $\sigma_{0}=\sqrt{\lambda^{2}+\frac{\mathrm{i} \omega}{D_{0}}}$ is the wave vector in the Hankel space, and $\omega$ is the pulsation of the pump beam. As a result, the temperature amplitude 
in real space is the convolution of the Gaussian beam by a spherical thermal wave $\frac{\exp \left(-r / \mu_{0}\right)}{r}$, where $\mu_{0}$ is the thermal diffusion length $\left(\mu_{0}=\sqrt{\frac{2 D_{0}}{\omega}}\right)$.

If a layer (thickness $h_{2}$, thermal conductivity $k_{2}$, and thermal diffusivity $D_{2}$ ) is deposited on the substrate (thermal conductivity $k_{0}$ and thermal diffusivity $D_{0}$ ), Eq. 1 becomes [9]

$$
T(r, 0, \omega)=\int_{0}^{\infty} \frac{Q}{k_{2} \sigma_{2}} \frac{k_{2} \sigma_{2}+k_{0} \sigma_{0} t h\left(h_{2} \sigma_{2}\right)}{k_{0} \sigma_{0}+k_{2} \sigma_{2} t h\left(h_{2} \sigma_{2}\right)} \exp \left(-\frac{\lambda^{2} d^{2}}{32}\right) J_{0}(\lambda r) \lambda \mathrm{d} \lambda .
$$

When an additional layer (thickness $h_{1}$, thermal conductivity $k_{1}$, and thermal diffusivity $D_{1}$ ) is present between the layer and the substrate, Eq. 2 becomes

$$
T(r, 0, \omega)=\int_{0}^{\infty} \frac{Q}{k_{2} \sigma_{2}} \frac{k_{2} \sigma_{2}+k_{0} \sigma_{0} \frac{\left[1+\frac{k_{1} \sigma_{1}}{k_{0} \sigma_{0}} \operatorname{th}\left(h_{1} \sigma_{1}\right)\right]}{\left[1+\frac{k_{0} \sigma_{0}}{k_{1} \sigma_{1}} \operatorname{th}\left(h_{1} \sigma_{1}\right)\right]} \operatorname{th}\left(h_{2} \sigma_{2}\right)}{k_{0} \sigma_{0} \frac{\left[1+\frac{k_{1} \sigma_{1}}{k_{0} \sigma_{0}} \operatorname{th}\left(h_{1} \sigma_{1}\right)\right]}{\left[1+\frac{k_{0} \sigma_{0}}{k_{1} \sigma_{1}} \operatorname{th}\left(h_{1} \sigma_{1}\right)\right]}+k_{2} \sigma_{2} \operatorname{th}\left(h_{2} \sigma_{2}\right)} \exp \left(-\frac{\lambda^{2} d^{2}}{32}\right) J_{0}(\lambda r) \lambda \mathrm{d} \lambda
$$

If we suppose that the intermediate layer is very thin $\left(h_{1} \ll h_{2}\right)$, that its thermal properties are very low compared to those of the substrate and layer $2\left(k_{1} \ll k_{2}, k_{0}\right)$ and if we note $R_{1}=\frac{h_{1}}{k_{1}}$ (the interface resistance), Eq. 3 is written as

$$
T(r, 0, \omega)=\int_{0}^{\infty} \frac{Q}{k_{2} \sigma_{2}} \frac{k_{2} \sigma_{2}+\frac{k_{0} \sigma_{0}}{1+R_{1} k_{0} \sigma_{0}} t h\left(h_{2} \sigma_{2}\right)}{\frac{k_{0} \sigma_{0}}{1+R_{1} k_{0} \sigma_{0}}+k_{2} \sigma_{2} t h\left(h_{2} \sigma_{2}\right)} \exp \left(-\frac{\lambda^{2} d^{2}}{32}\right) J_{0}(\lambda r) \lambda \mathrm{d} \lambda
$$

This expression demonstrates that, in the presence of an interface resistance $R_{1}$, we have just to replace $k_{0} \sigma_{0}$ by $\frac{k_{0} \sigma_{0}}{1+R_{1} k_{0} \sigma_{0}}$ in all the equations. Note that Eq. 4 can also be obtained directly by considering a temperature jump between the layer and the substrate proportional to the flux.

\section{Good Thermal Conductor Layer on Insulator Substrate}

In [10], we have established that the solution of Eq. 2 is achieved with the integration along a contour excluding the branch point associated with $\sigma_{0}$. The solution corresponds to a sum calculated for all the poles $\left(\lambda_{n}\right)$ and a term corresponding to the integration around the branch point:

$$
T(r, 0, \omega) \approx \sum A_{n} \frac{\exp \left(\mathrm{i} \lambda_{n} r\right)}{\sqrt{r}}+B \frac{\exp \left(-\varepsilon r \sqrt{\frac{\omega}{D_{0}}}\right)}{r}
$$

with $\varepsilon=\exp \left(\mathrm{i} \frac{\pi}{4}\right)$. In our case (good conductor on insulator), the second term is negligible except for extremely thin films or very low modulation frequencies. 
Approximations of the poles in Eq. 2 can be numerically found. However, in the case where the ratio $\alpha=\frac{k_{0}}{k_{2}}$ is small, they can then be found by expanding the denominator $\alpha \sigma_{0}+\sigma_{2} t h\left(h_{2} \sigma_{2}\right)$ in a power series of this parameter. The asymptotic behavior corresponds to the pole which exhibits the smallest imaginary part:

$$
\left.\lambda_{0}^{2}=-\mathrm{i} \frac{\omega}{D_{2}}-\varepsilon \alpha \sqrt{\frac{\omega}{D_{0}}-\frac{\omega}{D_{2}}} \text { (up to the first order in } \alpha\right)
$$

It is then straightforward that the asymptotic lateral heat diffusion corresponds to a radial-plate-like regime with a complex diffusion length:

$$
\begin{aligned}
& T(\infty, 0, \omega) \approx \frac{\exp \left(\mathrm{i} r \sqrt{\lambda_{0}^{2}}\right)}{\sqrt{r}}=\frac{\exp (-r(a+\mathrm{i} b))}{\sqrt{r}} \\
& \text { with } h\left(a^{2}-b^{2}\right)=\frac{k_{0}}{k_{2}} \frac{\sqrt{\omega}}{\sqrt{2}} \sqrt{\frac{1}{D_{0}}-\frac{1}{D_{2}}} \approx \frac{k_{0}}{k_{2}} \frac{\sqrt{\omega}}{\sqrt{2}} \sqrt{\frac{1}{D_{0}}} \\
& \text { and }\left(b^{2}-a^{2}+2 a b\right)=\frac{\omega}{D_{2}}
\end{aligned}
$$

When an interface resistance is suspected, we have to replace $k_{0} \sigma_{0}$ with $\frac{k_{0} \sigma_{0}}{1+R_{1} k_{0} \sigma_{0}}$ in the denominator of Eq. 2. In the following, we consider that the presence of the interface resistance is a perturbation of the previous system.

The poles are now calculated with the perturbed equation:

$$
\alpha \sigma_{0}\left(1-R_{1} k_{0} \sigma_{0}\right)+\sigma_{2} t h\left(h_{2} \sigma_{2}\right)=0 \text {. }
$$

The asymptotic behavior corresponds again to the pole which exhibits the smallest imaginary part:

$\lambda_{0 R}^{2}=-\mathrm{i} \frac{\omega}{D_{2}}\left(1+R_{1} \frac{k_{0}^{2}\left(\frac{\omega}{D_{2}}-\frac{\omega}{D_{0}}\right)}{h_{2} k_{2} \frac{\omega}{D_{2}}}\right)-\varepsilon \alpha \sqrt{\frac{\omega}{D_{0}}-\frac{\omega}{D_{2}}}$ (up to the first order in $\left.\alpha\right)$.

The asymptotic lateral heat diffusion corresponds again to a radial-plate-like regime:

$$
\begin{aligned}
& T(\infty, 0, \omega) \approx \frac{\exp \left(\mathrm{i} r \sqrt{\lambda_{0 R}^{2}}\right)}{\sqrt{r}}=\frac{\exp \left(-r\left(a_{R}+\mathrm{i} b_{R}\right)\right)}{\sqrt{r}} \\
& \text { with } h_{2}\left(a_{R}^{2}-b_{R}^{2}\right)=\frac{k_{0}}{k_{2}} \frac{\sqrt{\omega}}{\sqrt{2}} \sqrt{\frac{1}{D_{0}}-\frac{1}{D_{2}}} \approx \frac{k_{0}}{k_{2}} \frac{\sqrt{\omega}}{\sqrt{2}} \sqrt{\frac{1}{D_{0}}}=p_{R} \sqrt{\omega} \\
& \left(b_{R}^{2}-a_{R}^{2}+2 a_{R} b_{R}\right)=\frac{\omega}{D_{2}}+R_{1} \omega \frac{k_{0}^{2}}{h_{2} k_{2}}\left(\frac{1}{D_{2}}-\frac{1}{D_{0}}\right) \approx \frac{\omega}{D_{2}}-\frac{R_{1} \omega k_{0}^{2}}{h_{2} k_{2} D_{0}}=q_{R} \omega .
\end{aligned}
$$


A comparison of Eqs. 8 and 9 and Eqs. 13 and 14 shows that the measurement of the thermal conductivity is not affected by the presence of the interface resistance while the effective thermal-diffusivity measurement $\left(1 / q_{R}\right)$ has to be corrected to find the thermal diffusivity $D_{2}$ and $R_{1}$.

Figure 1a, b shows the experimental data for five modulation frequencies, for a $55 \mathrm{~nm}$ thick aluminum layer deposited on fused silica. The thermal conductivity and
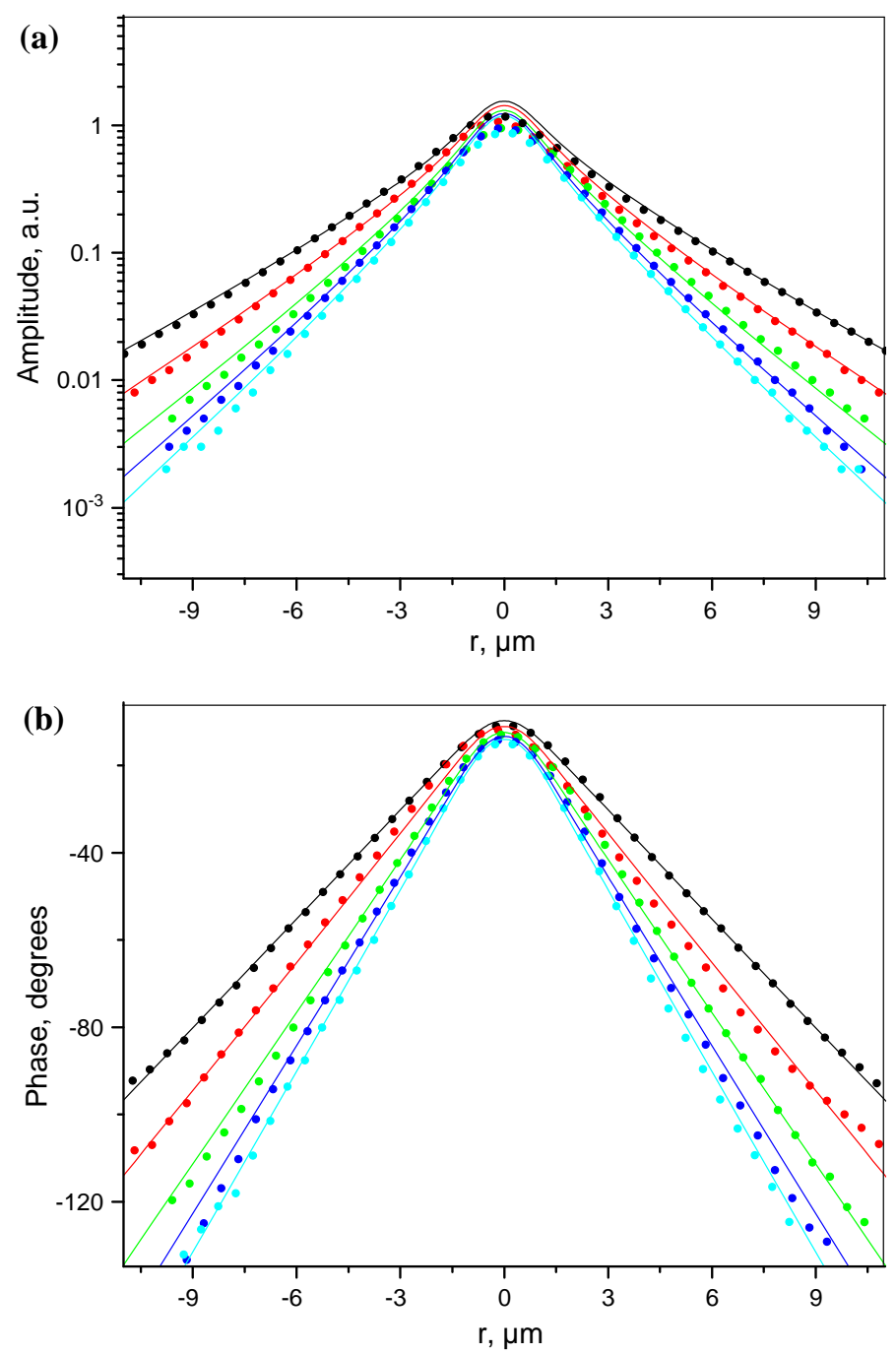

Fig. 1 Thermoreflectance microscope (a) amplitude and (b) phase signal for $55 \mathrm{~nm}$ thick Al layer on a semi-infinite fused silica substrate, recorded at five frequencies $(75,150,300,450$, and 600) kHz. Symbols experimental points. Lines fits obtained with effective values (thermal diffusivity-3.45 $\times 10^{-4} \mathrm{~m}^{2} \cdot \mathrm{s}^{-1}$ and thermal conductivity- $154 \mathrm{~W} \cdot \mathrm{m}^{-1} \cdot \mathrm{K}^{-1}$ ) or with the corrected values (thermal diffusivity$0.6 \times 10^{-4} \mathrm{~m}^{2} \cdot \mathrm{s}^{-1}$, thermal conductivity $-154 \mathrm{~W} \cdot \mathrm{m}^{-1} \cdot \mathrm{K}^{-1}$, and $R_{i}-5 \times 10^{-8} \mathrm{~m}^{2} \cdot \mathrm{K} \cdot \mathrm{W}^{-1}$ ). Both sets of lines are superimposed 
the thermal diffusivity of the layer were deduced from $p$ and $q$ values (Eqs. 8, 9) because the thermal properties of the substrate and the thickness of the layer are known. We can remark that the thermal conductivity $\left(154 \mathrm{~W} \cdot \mathrm{m}^{-1} \cdot \mathrm{K}^{-1}\right)$ is smaller than the thermal conductivity of a bulk aluminum sample $\left(237 \mathrm{~W} \cdot \mathrm{m}^{-1} \cdot \mathrm{K}^{-1}\right)$, while the thermal diffusivity $\left(3.45 \times 10^{-4} \mathrm{~m}^{2} \cdot \mathrm{s}^{-1}\right)$ is larger $\left(0.95 \times 10^{-4} \mathrm{~m}^{2} \cdot \mathrm{s}^{-1}\right)$. It is well known that the value of the thermal conductivity decreases when the thickness of the layer decreases, but the value of the thermal diffusivity is largely overestimated. Aluminum deposition presents a very small adhesion, and an interface layer is likely. Equations 13 and 14 should then be used to determine the thermal properties of the layer and to evaluate the thermal resistance. The thermal conductivity being determined with Eq. 13, a common hypothesis is to keep the ratio $k_{2} / D_{2}$ constant [11], which is equivalent to the reasonable assumption that the volumetric heat capacity is a material constant. So, it is possible to know the value of the interface resistance from Eq. 14. The thermal properties of the layer are estimated to be: $k_{2}=154 \mathrm{~W} \cdot \mathrm{m}^{-1} \cdot \mathrm{K}^{-1}$ and $D_{2}=0.6 \times 10^{-4} \mathrm{~m}^{2} \cdot \mathrm{s}^{-1}$ and the value of the interface thermal resistance to be $R_{1}$ $=5 \times 10^{-8} \mathrm{~m}^{2} \cdot \mathrm{K} \cdot \mathrm{W}^{-1}$.

In conclusion, when an interface resistance is present (weak adhesion, for instance) the heat is more confined in the layer than expected and a high value of the thermal diffusivity is deduced from the "reduced" model of the lateral heat diffusion. In this case, the second model taking into account the resistance has to be used.

\section{Poor Thermal Conductor Layer on Good Thermal Conductor Substrate}

In this case, layer 2 affects the spatio-temporal temperature field mainly around the heat spot [12]. The lateral diffusion being weak in the layer, a spherical thermal wave is developed in the substrate which heats the surface through the thin layer far from the center. In this case, the contribution of the layer to the lateral heat diffusion is a perturbation with respect to the substrate one. If the thickness of the layer is small, Eq. 2 can be developed:

$$
\begin{aligned}
& T(r, 0, \omega)=\int Q\left[\frac{1}{k_{0} \sigma_{0}}+\frac{t h\left(h_{2} \sigma_{2}\right)}{k_{2} \sigma_{2}} \frac{1-\left(\frac{k_{2} \sigma_{2}}{k_{0} \sigma_{0}}\right)^{2}}{1+\frac{k_{2} \sigma_{2}}{k_{0} \sigma_{0}} t h\left(h_{2} \sigma_{2}\right)}\right] \exp \left(-\frac{\lambda^{2} d^{2}}{32}\right) J_{0}(\lambda r) \lambda \mathrm{d} \lambda \\
& T(r, 0, \omega)=\int Q\left[\frac{1}{k_{0} \sigma_{0}}+\frac{h_{2}}{k_{2}}\left(1-\left(\frac{k_{2} \sigma_{2}}{k_{0} \sigma_{0}}\right)^{2}\right)\right] \exp \left(-\frac{\lambda^{2} d^{2}}{32}\right) J_{0}(\lambda r) \lambda \mathrm{d} \lambda
\end{aligned}
$$

Up to the first order, the contribution of the layer is linear with respect to $h_{2}$. When an interface resistance is suspected, we have just to replace $\frac{1}{k_{0} \sigma_{0}}$ by $\frac{1}{k_{0} \sigma_{0}}+R_{1}$ in Eq. 16 . Then, the following equation has to be taken into consideration: 

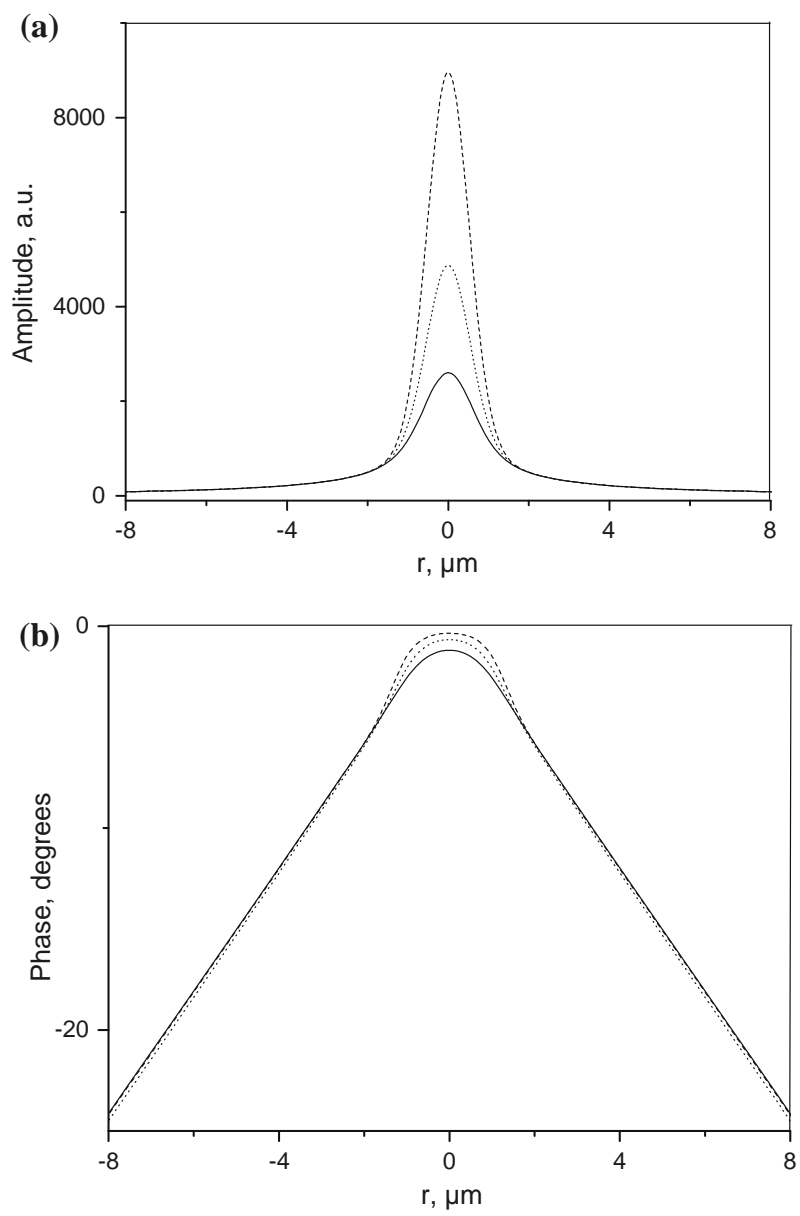

Fig. 2 Calculated thermoreflectance microscope (a) amplitude and (b) phase signal. Influence of an interface resistance of $10^{-8} \mathrm{~m}^{2} \cdot \mathrm{K} \cdot \mathrm{W}^{-1}$ between a $80 \mathrm{~nm}$ Ti layer and a silicon substrate at $80 \mathrm{kHz}$ modulation frequency. The equivalent resistance value of the Ti film is Req $=4 \times 10^{-9} \mathrm{~m}^{2} \cdot \mathrm{K} \cdot \mathrm{W}^{-1}$. Dotted line Ti layer on silicon without interface resistance. Dashed line Ti layer on silicon with the interface resistance. Full line bare silicon substrate

$$
\begin{aligned}
T(r, 0, \omega) \approx & \int Q\left[\frac{1}{k_{0} \sigma_{0}}+R_{1}+\frac{h_{2}}{k_{2}}\left(1-\frac{k_{2}^{2} \sigma_{2}^{2}\left(1+2 R_{1} k_{0} \sigma_{0}\right)}{k_{0}^{2} \sigma_{0}^{2}}\right)\right] \\
& \exp \left(-\frac{\lambda^{2} d^{2}}{32}\right) J_{0}(\lambda r) \lambda \mathrm{d} \lambda .
\end{aligned}
$$

An interface resistance behaves always as an insulator layer, but in this case this is apparent in the mathematical expression of Eq. 17. Its contribution appears mainly near the heating spot and disrupts the perpendicular diffusion scheme (Fig. 2a, b). In a previous article [12], we have studied the behavior of asymptotic lateral diffusion and demonstrated that an insulating layer deposited on a good conductor affects very 
slightly the heat lateral diffusion. The presence of a thermal resistance will have the same effect, and it is practically not possible to extract its value from the lateral behavior of the heat.

\section{Conclusion}

The presence of an interface resistance affects differently the behavior of the heat lateral diffusion depending on the contrast of thermal properties of the substrate and the layer. In the case of a good conductor deposited on an insulator, the heat is more confined in the layer than expected and an overestimated value of the thermal diffusivity is deduced from the "reduced" model of the lateral heat diffusion. This is a "thermal signature" of the thermal resistance. In this case, a model which takes into account the resistance has to be used, and it is possible to obtain both the thermal properties of the layer and the value of the thermal resistance. In the opposite case (insulator on good conductor), there is little evidence of the presence of the thermal resistance on the lateral behavior of the heat diffusion. The interface resistance affects clearly the perpendicular diffusion, and its presence can be seen only close to the heating spot.

\section{References}

1. B.C. Gundrum, D.G. Cahill, R.S. Averback, Phys. Rev. B 72, 245426 (2005)

2. H.-K. Lyeo, D.G. Cahill, Phys. Rev. B 76, 144301 (2006)

3. D.L. Balageas, J.C. Krapez, P. Cielo, J. Appl. Phys. 59, 348 (1986)

4. J.-L. Battaglia, A. Kusiak, V. Schick, J. Appl. Phys. 107, 044314 (2010)

5. D.G. Cahill, Rev. Sci. Instrum. 61, 802 (1990)

6. A. Rosencwaig, J. Opsal, W.L. Smith, D.L. Willenborg, Appl. Phys. Lett. 46, 1013 (1985)

7. L. Pottier, Appl. Phys. Lett. 64, 1618 (1994)

8. B. Li, J.-P. Roger, L. Pottier, D. Fournier, J. Appl. Phys. 86, 5314 (1999)

9. K. Plamann, D. Fournier, B.C. Forget, A.C. Boccara, Diamond Relat. Mater. 5, 699 (1996)

10. C. Frétigny, J.P. Roger, V. Reita, D. Fournier, J. Appl. Phys. 102, 116104 (2007)

11. G. Langer, J. Hartmann, M. Reichling, Rev. Sci. Intrum. 68, 1510 (1997)

12. C. Frétigny, J.-Y. Duquesne, D. Fournier, F. Xu, J. Appl. Phys. 111, 084313 (2012) 